\title{
Online resources for data exploration, visualization, and discovery for the Pan-Cancer Analysis of Whole Genomes project (PCAWG)
}

Mary Goldman*1, Junjun Zhang ${ }^{* 2}$, Nuno A. Fonseca*3, Isidro Cortés-Ciriano*4,5, Qian Xiang ${ }^{2}$, Brian Craft ${ }^{1}$, Elena Piñeiro-Yáñez ${ }^{6}$, Brian D O'Connor ${ }^{1}$, Wojciech Bazant ${ }^{3}$, Elisabet Barrera ${ }^{3}$, Alfonso Muñoz-Pomer ${ }^{3}$, Robert Petryszak ${ }^{3}$, Anja Füllgrabe ${ }^{3}$, Fatima Al-Shahrour ${ }^{6}$, Maria Keays ${ }^{3}$, David Haussler ${ }^{1}$, John N. Weinstein ${ }^{7}$, Wolfgang Huber ${ }^{8}$, Alfonso Valencia ${ }^{9,10}$, Peter J. Park ${ }^{4}$, Irene Papatheodorou\# ${ }^{3}$, Jingchun Zhu\# ${ }^{1}$, Vincent Ferretti ${ }^{11}$, Miguel Vazquez\# $\#^{9,12}$, on behalf of the PCAWG Portals and Visualization Working Group, and the ICGC/TCGA Pan-Cancer Analysis of Whole Genomes Network

${ }^{1}$ UC Santa Cruz Genomics Institute, Santa Cruz, 95064, USA.

${ }^{2}$ Ontario Institute for Cancer Research, Toronto, ON M5G 0A3, Canada.

${ }^{3}$ European Molecular Biology Laboratory, European Bioinformatics Institute, EMBL-EBI, Hinxton, CB10 1SD, UK.

${ }^{4}$ Department of Biomedical Informatics, Harvard Medical School, Boston, MA, USA.

${ }^{5}$ Centre for Molecular Science Informatics, Department of Chemistry, University of Cambridge, Lensfield Road, Cambridge CB2 1EW, United Kingdom.

${ }^{6}$ Bioinformatics Unit, Spanish National Cancer Research Centre (CNIO), Madrid, 28029, Spain

${ }^{7}$ UT MD Anderson Cancer Center, Houston, 77030, USA.

${ }^{8}$ European Molecular Biology Laboratory, Heidelberg, 69117, Germany.

${ }^{9}$ Barcelona Supercomputing Center (BSC), Barcelona, 08034, Spain.

${ }^{10}$ ICREA, Barcelona, 08010, Spain.

${ }^{11}$ CHU Sainte-Justine Research Center, Montreal, Quebec, H3T 1C5, Canada.

${ }^{12}$ Norwegian University of Science and Technology, Trondheim, Norway.

\section{Abstract (150 words)}

Pan-Cancer Analysis of Whole Genomes (PCAWG) encompasses the largest collection, to date, of uniformly-processed, cancer whole-genome sequencing data sets. While this dataset has the power to elucidate many cancer biology mechanisms, it also poses serious challenges for visualization tools because of its size and complexity. Here we describe five publicly available tools, each either designed or augmented to derive insights from this unique resource: The ICGC Data Portal, UCSC Xena, Expression Atlas, PCAWG-Scout, and Chromothripsis Explorer. Each of these web tools is designed to be user-friendly, putting sophisticated analyses in the hands of all researchers. Together, they enable researchers to dynamically query these complex genomics data, explore tumors' molecular landscapes, and integrate external information to facilitate data interpretation. More information on these tools and their capabilities is available from The PCAWG Landing Page (http://docs.icgc.org/pcawg).

\section{Main text}

The Pan-Cancer Analysis of Whole Genomes (PCAWG) cohort provides the largest uniformlyanalyzed, publicly-available whole-genome dataset in cancer genomics. The PCAWG project has reprocessed whole-genome sequencing data for 2,658 donors from 48 cancer projects across 47 different primary tumor types. This reprocessing involved alignment to the reference genome, quality 
assessment, identification of somatic and germline variants, and generation of consensus mutation calls derived from multiple bioinformatics methods (Campbell 2017, Yung 2017). While this dataset is rich in biological insights into cancer, visualization is challenging due to the large number of whole genomes. First, many visualization tools that focus on genes do not have a way to explicitly view the intergenic and intronic regions that whole genome sequencing elucidates. Second, the large size of whole-genome data imposes high performance requirements for interactive tools, especially those on the web. Finally, the analysis of whole-genome data provides an array of high quality genomic information, including point mutations, gene fusions, promoter usage, and structural variants across thousands of samples and dozens of disease entities. Many current visualization tools are not equipped to be able to take advantage of depth and complexity of information at each point of the genome.

Five biologist-friendly tools were created or extended to address these challenges as part of the PCAWG project: The ICGC Data Portal, UCSC Xena, Chromothripsis Explorer, Expression Atlas, and PCAWG-Scout. Each tool has taken steps to ensure that it is easy-to-use, including making the tool accessible through a web interface so that no extra software is needed, pre-loading the PCAWG data so that users do not need to locate or manage data, and specifically designing the website/interface for users to easily configure visualizations and analyses. In addition to ease-of-use, these five tools also focused on integrating other genomics datasets and tools that provide insights into genomic patterns within the PCAWG data. Some of these datasets include the UCSC Genome Browser (Kent 2002), Ensembl (Zerbino 2018), drug target compendia (Piñeiro-Yáñez 2018), and large sequencing efforts such as GTEx (Carithers 2015). The five tools described here provide intuitive access to these resources, either by showing the data from the resources side-by-side or providing context-dependent URL links, making it easy for researchers to contextualize findings from the PCAWG resource.

Researchers interested in these tools can learn about and access them at the PCAWG Landing Page (http://docs.icgc.org/pcawg).

The ICGC Data Portal serves as the main entry point for accessing all PCAWG data and also can be used to explore PCAWG consensus simple somatic mutations, including point mutations and small indels, each by their frequencies, patterns of co-occurrence, mutual exclusivity, and functional associations. UCSC Xena integrates diverse types of genomic and phenotypic/clinical information at the sample-level across large number of samples, enabling rapid examination of patterns within and across data types. The Chromothripsis Explorer visualizes genome-wide mutational patterns, with a focus on complex events, e.g., chromothripsis and kataegis. This is done through interactive Circos plots for each tumor with different tracks corresponding to allele-specific copy number variants, somatic structural variations, simple somatic mutations, indels, and clinical information. The Expression Atlas focuses on RNA-seq data, supporting queries in either a baseline context (e.g., find genes that are expressed in prostate adenocarcinoma samples) or in a differential context (e.g., find genes that are under- or over-expressed in prostate adenocarcinomas compared to "adjacent-normal" prostate samples). PCAWG-Scout allows users to run their own analysis on-demand, including predicting cancer driver genes, differential gene expression, calling recurrent structural variations, survival analysis, pathway enrichment, visualization of mutations on a protein structure, analysis of mutational signatures, and predictions of recommended therapies based on their in-house resource, 
PanDrugs (Supplementary Figure 1). Each of these five tools offers complementary views of the PCAWG data resource, providing deep biological insights (Figure 1). Together these resources enable biologists to dynamically query and integrate the complex genomics data, explore tumors' molecular landscapes, and combine external data with the PCAWG cohort to facilitate interpretation (Table 1).

\section{PCAWG data and access}

These five resources support the primary 'omic data types generated by the PCAWG project, including simple somatic mutations (single- and multiple- nucleotide variants (SNVs, MNVs)); small insertions and deletions (INDELs); large somatic structural variants (SVs); copy number variants; gene fusions; RNA-seq gene- and miRNA-expression; DNA methylation; and phenotypic annotations (Li 2017, PCAWG Transcriptome Core Group 2018, Rheinbay 2017, Sabarinathan 2017, Yung 2017). Two types of files were generated by the PCAWG analysis: primary BAM and VCF files, and downstream analysis results (Campbell 2017). The ICGC Data Portal provides a uniform search interface for both file types (https://dcc.icgc.org). Each of the four other resources, UCSC Xena, Chromothripsis Explorer, Expression Atlas, and PCAWG-Scout, separately ingested the same primary result files and individually refined them for online visualization, exploration and download (Fig. 1A, Supplementary Table 1, Supplementary Figure 2).

Access to protected data (i.e., primary BAM and VCF files, germline variant calls, and simple somatic mutation calls for non-coding regions) is provided through the ICGC Data Portal for researchers with approved authorization (for instructions for how to gain access please see https://docs.icgc.org/pcawg/data/\#apply-for-data-access). UCSC Xena provides secure views of protected, non-coding somatic mutations through the use of a private Xena data hub. Both the ICGC Data Portal and PCAWG-Scout publicly display non-identifiable analysis results of protected data.

\begin{tabular}{|c|c|c|c|c|c|c|}
\hline \multicolumn{2}{|r|}{ Functionality } & $\begin{array}{l}\text { ICGC } \\
\text { Data }\end{array}$ & $\begin{array}{l}\text { UCSC } \\
\text { Xena }\end{array}$ & $\begin{array}{l}\text { Chromo- } \\
\text { thripsis }\end{array}$ & $\begin{array}{l}\text { Expression } \\
\text { Atlas }\end{array}$ & $\begin{array}{l}\text { PCAWG } \\
\text {-Scout }\end{array}$ \\
\hline \multirow{3}{*}{$\begin{array}{l}\frac{c}{0} \\
\frac{0}{\sigma} \\
\Phi \\
\text { ஸे }\end{array}$} & $\begin{array}{l}\text { Search by demographic data, specimen } \\
\text { phenotype, molecular subtype }\end{array}$ & Y & Y & & & $\mathrm{Y}$ \\
\hline & Search by genes and/or variants & Y & $\mathrm{Y}$ & & Y & Y \\
\hline & Search by genomic coordinates & Y & $\mathrm{Y}$ & & & \\
\hline \multirow{5}{*}{ 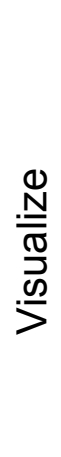 } & Visualize multiple types of data together & & $\mathrm{Y}$ & $\mathrm{Y}$ & Y & $\mathrm{Y}$ \\
\hline & Visualize coding variants & $\mathrm{Y}$ & $\mathrm{Y}$ & $\mathrm{Y}$ & & $\mathrm{Y}$ \\
\hline & Visualize non-coding variants & $\mathrm{Y}$ & $\mathrm{Y}$ & $\mathrm{Y}$ & & \\
\hline & Visualize structural variants & & Y & $\mathrm{Y}$ & & $\mathrm{Y}$ \\
\hline & $\begin{array}{l}\text { Visualize mutational signatures and predicted } \\
\text { drivers }\end{array}$ & & & & & $\mathrm{Y}$ \\
\hline
\end{tabular}




\begin{tabular}{|c|c|c|c|c|c|c|}
\hline & $\begin{array}{l}\text { Visualize genome-wide profiles, including } \\
\mathrm{LOH} \text {, in Circos plots }\end{array}$ & & & $\mathrm{Y}$ & & \\
\hline & Visualize tissue expression on a human figure & & & & $\mathrm{Y}$ & \\
\hline & Visualize gene co-expression & & Y & & Y & \\
\hline & Visualize pathways, therapeutic associations & Y & & & & Y \\
\hline & Visualize summary of BAMs/VCFs & Y & & & & \\
\hline & Kaplan-Meier analysis with statistics & Y & Y & & & Y \\
\hline & Geneset/pathway enrichment analysis & Y & & & Y & Y \\
\hline$\frac{\infty}{\infty}$ & $\begin{array}{l}\text { View non-identifiable analysis results of } \\
\text { protected data }\end{array}$ & Y & & Y & Y & Y \\
\hline 这 & $\begin{array}{l}\text { Discover differentially- or co-expressed } \\
\text { genes, mutually exclusive genomic events }\end{array}$ & & & & & Y \\
\hline & Annotations from other resources & $\mathrm{Y}$ & $\mathrm{Y}$ & & Y & Y \\
\hline & Programmatic data download & $\mathrm{Y}$ & $\mathrm{Y}$ & & Y & Y \\
\hline 읃 & Download BAMs, VCFs, primary files & $\mathrm{Y}$ & & & & \\
\hline คำ & Download secondary processed data & $Y$ & $\mathrm{Y}$ & & $Y$ & $Y$ \\
\hline
\end{tabular}

Table 1. Search, visualization, analysis/integration, and download functionalities provided by each of the PCAWG data resources.

ICGC Data Portal (https://dcc.icgc.org). As a main entry point, the ICGC Data Portal provides an intuitive graphic interface for users to browse, search and explore PCAWG data sets (Figure 1A). Uniformly aligned sequencing BAM files and variant calling VCF files, although physically residing in multiple repositories globally, can be centrally searched via a faceted search interface (https://icgc.org/ZEA). Users can easily find specific data sets of interest with a few mouse clicks. A universal data access tool called "icgc-get" can be used to download data from different repositories. Other downstream analysis results generated by PCAWG working groups are available at https://dcc.icgc.org/releases/PCAWG. Close to 23 million open access PCAWG consensus simple somatic mutations have been annotated with consequences in protein changes, affected pathways, targeting cancer drugs and gene ontology terms alongside with clinical information integration. The portal's Advanced Search (https://icgc.org/ZzP) tool allows users to perform complex queries, such as, retrieving the most frequently mutated genes that are targets of drugs from stage 2 liver cancer patients (https://icgc.org/ZHe). Analytic tools (https://dcc.icgc.org/analysis) support exploration of potential associations between molecular abnormalities and phenotypic observations such as patient survival. 
UCSC Xena (https://pcawg.xenahubs.net). UCSC Xena's adaptable visualizations, fast performance, and flexible data format bring the full power of the PCAWG resource to all researchers. UCSC Xena is a tool to explore functional cancer genomics data (Goldman 2018) geared towards cancer researchers. It displays data mapped to coding and non-coding regions of the genome, including introns, promoters, enhancers, and intergenic regions. Xena can display tens of thousands of data points on thousands of samples, all within seconds. Xena visualizes a comprehensive list of PCAWG primary and derived results, such as gene expression, copy number variation, simple mutation, phenotypic data, miRNA expression, coding driver mutations, alternative splicing events, gene fusions, promoter usage, large somatic structural variation, mutational signatures, APOBEC mutagenesis analysis, purity, and ploidy ('PCAWG hub', Supplementary Table 2). The Xena Browser excels at integrating these diverse datasets using the Visual Spreadsheet (Figure 1B), allowing users to view different types of data side-by-side. In addition to the Visual Spreadsheet, Xena's KaplanMeier plots, histograms, boxplots, scatterplots, dynamic subgrouping, and bookmarks offer additional views and statistical analyses.

Xena's hub-browser architecture enables users to view the protected consensus simple somatic mutations (including non-coding mutations) by loading the dataset into a user's local private Xena hub (Figure 2, Supplementary Figure 3). The Xena Browser seamlessly integrates data from multiple hubs, allowing users who have access to the protected mutation data to visualize it in conjunction with other PCAWG data publicly available on the PCAWG Xena Hub (https://pcawg.xenahubs.net).

Chromothripsis Explorer (http://compbio.med.harvard.edu/chromothripsis/). Chromothripsis refers to the mutational process characterized by massive de novo rearrangements that affect one or multiple chromosomes (Stephens 2011). The whole-genome data set assembled by PCAWG now provides an unparalleled opportunity to characterize chromothripsis patterns on a large-scale at single-base resolution across over 30 cancer types. Although statistical metrics are generally used to identify chromothripsis patterns (Korbel 2013), visual inspection still remains essential to dismiss false positive cases generated by other mechanisms of genome instability (Cortes-Ciriano 2018; Notta 2016). The Chromothripsis Explorer is an open source $R$ Shiny application that visualizes chromothripsis patterns, allele-specific copy number alterations, purity and ploidy estimates, SNVs, indels, and structural variants detected using WGS data (Cortes-Ciriano 2018; Campbell 2017). The R Shiny application can be downloaded and installed locally (https://github.com/parklab/ShatterSeek).

The Chromothripsis Explorer provides tools for the exploration of chromothripsis frequencies and patterns across tumor types (Figure 3a). Specifically, it provides interactive Circos plots (Yu 2018) for each tumor, allowing researchers to explore large-scale alterations such as chromosome arm deletions, and complex mutational patterns such as chromothripsis and chromoplexy (Figure 3b). Each Circos plot is divided into 7 tracks that display, from outer to inner rings: (i) hg19 cytobands; (ii) inter-mutation distance and location for pathogenic (i.e., non-synonymous, stop-gain, and stop-loss) and nonpathogenic SNVs, and frame-shift and in-frame indels; (iii) chromothripsis regions; (iv) total copy number; ( $v$ ) minor copy number profiles, defined as the least amplified allele, to visualize LOH regions; and (vi) gene track, and (vii) structural variations displayed according to the read orientations at the breakpoints (duplication-like SVs in blue, deletion-like SVs in orange, head-to-head in black, 
and tail-to-tail inversions in green). By hovering over the Circos plots, one can obtain information about a mutation of interest at single-base resolution, as well as gene annotations and functional effect predictions. In addition to genomics data, clinical and histo-pathological data are provided for all tumors in the form of customizable tables that permit to map tumor identifiers across cancer projects (e.g., TCGA to ICGC IDs; Figure 3c).

Expression Atlas (https://www.ebi.ac.uk/gxa/home). Expression Atlas is an added-value database and web-service that enables users to identify gene expression in different tissues, cell types, diseases and developmental stages. It collects, annotates, re-analyses and displays gene, transcript and protein expression data. It supports two types of study design: baseline and differential. Baseline studies involve quantifications of genes within tissues, developmental stages, cell lines, as well as others. Differential studies perform differential expression comparisons between different samples, for example disease versus healthy tissues (Fig. 4). In addition to the PCAWG datasets, expression studies from archives such as ArrayExpress, GEO (Gene Expression Omnibus) and ENA (European Nucleotide Archive) are selected for further curation and processing. Data curation involves a semiautomated process of identifying the experimental factors, such as diseases or perturbations, annotating metadata with Experimental Factor Ontology terms (EFO) and describing the experimental comparisons for further processing. Currently, we provide results on over 3,300 experiments that include about 120,000 assays from over 40 different organisms. The data sets cover over 100 cell types from the Cell Ontology and over 700 diseases represented in the EFO.

Expression Atlas includes large landmark baseline studies on human subjects or cell lines, such as GTEx, CCLE, ENCODE, BLUEPRINT, HipSci, as well as differential studies on human diseases in human patients or animal models. Analyses of bulk or single cell RNA-seq data sets are performed using our open source pipeline iRAP (https://nunofonseca.github.io/irap). Expression Atlas can be searched by gene, gene set and experimental condition queries (Figure 4a). Gene, transcript and protein expression across different conditions is displayed through heatmaps and boxplots (Figure 4b). Annotation of data sets with EFO terms enables nested searching across related tissues, diseases and other conditions modeled within EFO. For example, when searching for "cancer", the search will produce results for all different types of cancer, including "leukemia".

PCAWG-Scout (http://pcawgscout.bsc.es/). As opposed to only offering a limited and predefined list of analyses, PCAWG-Scout offers a variety of on-demand analysis functionalities. These analyses allow researchers to explore and visualize the data, form a hypothesis, run the relevant analysis, and immediately explore and visualize the results, giving rise to an analysis loop that drives discovery. These analyses are performed on data from the PCAWG main data release, as available in the ICGC data repository, and results from the different working groups. The structure of the web interface accommodates reports for single entities (e.g. cohorts, samples, and genes) and lists of entities. These reports show relevant information about the entities, such as descriptions, statistics, plots, or interactive 3D protein representations and network graphs (Figure 5). Reports also offer further analyses that can be performed over the entities, such as enrichment analysis of gene lists, driver predictions over cohorts, survival analysis for lists of donors, or (non-authoritative) recommended therapies for individual donors (Supplementary Figure 4). The user can navigate these reports and analysis results as well as instantiate new reports as desired. The web interface uses a plugin 
approach to extend the reports and analysis that can be performed, making it easy for advanced users to add additional analyses. To help integrate PCAWG-Scout in a larger context, data and results are exported in interoperable formats.

Case study We demonstrate the value each resource brings to the PCAWG data using a common driver event in prostate cancer: the fusion of the oncogene ERG (St. John 2012, Adamo 2016). Looking across all 18 PCAWG prostate donor samples with both whole-genome sequencing and RNA-seq data, Xena's visual spreadsheet shows that 8 of them harbor an ERG fusion. These donors also show ERG over-expression (Figure 1b). A view of the PCAWG structural variant data shows that across all donors, the fusion breakpoints are located at the start of ERG, leaving the ERG coding region intact while fusing to the promoter region of TMPRSS2 or SLC45A3 (Figure 1b).

The Chromothripsis Explorer shows that CNV and SV alterations in the 8 tumors with ERG fusions vary widely (Figure 1c and Supplementary Figure 5). While donors DO36372, DO36359, DO36265 and D036335 have quiescent genomes with few SVs, DO36356 and D036283 show more complex karyotypes. For instance, patient DO36345 harbors a chain of balanced rearrangements reminiscent of chromoplexy, a type of complex event characterized by balanced translocations across multiple chromosomes (Baca 2013).

Both TMPRSS2 and SLC45A3 are highly expressed in normal prostate tissues and prostate tumors, as shown in the Expression Atlas Baseline Expression Widget (Fig. 1d). Combined analysis of the PCAWG and GTEx datasets leads to the hypothesis that a subset of prostate cancers, through genome rearrangement, hijack the promoters of androgen-responsive genes to increase ERG expression, resulting in an androgen-dependent over-expression of ERG.

Although the ERG fusions are frequent, 46\% (89 out of 195) of the PCAWG prostate tumors do not show them (Supplementary Figure 6). PCAWG-Scout's mutual exclusivity analysis shows that simple mutations in FOXA1, SPOP, SYNE1, and ANKFN1 are significantly associated with these non-fusion tumors (Figure 1e). Furthermore, in PCAWG-Scout's 3D protein structure view, the mutations in SPOP are shown to cluster tightly around the interaction interface for PTEN (Figure 1e), suggesting that those mutations may lead to altered protein function for SPOP.

Discussion The PCAWG resource is a deep and powerful resource for examining cancer biology. Here we have described five tools that are user-friendly, available online, and address many of the challenges of visualizing and integrating whole-genome data. These tools are The ICGC Data Portal, UCSC Xena, Expression Atlas, PCAWG-Scout, and Chromothripsis Explorer, all of which are available from The PCAWG Landing Page (http://docs.icgc.org/pcawg).

In addition to these five resources, there are additional online tools from the PCAWG consortium. One of these shows the panorama of driver mutations in PCAWG tumors that can be explored via Gitools interactive heatmaps (http://www.gitools.org/pcawg), and browsed in IntOGen, at http://www.intogen.org/pcawg (Sabarinathan 2017). We envision the PCAWG data will be incorporated into many other existing tools, and new resources will be developed. 
In addition to providing online resources for PCAWG data exploration, our tools also support opensource science. We have made the code for each resource publicly available (Supplementary Table 3) as well as developed embeddable Javascript modules for ICGC's OncoGrid, UCSC Xena's Visual Spreadsheet, and Expression Atlas's Heatmap and Anatomogram Widget (Supplementary Table 4). The licenses for these modules allow their incorporation into 3rd party web applications.

Our tools bring the deep and unusual datasets of the PCAWG consortium to the science community in five easy-to-use tools for visualization and analysis. These resources facilitate exploration of the PCAWG datasets and enable both bench biologists and bioinformaticians to gain insights from the data and make new discoveries. 
a

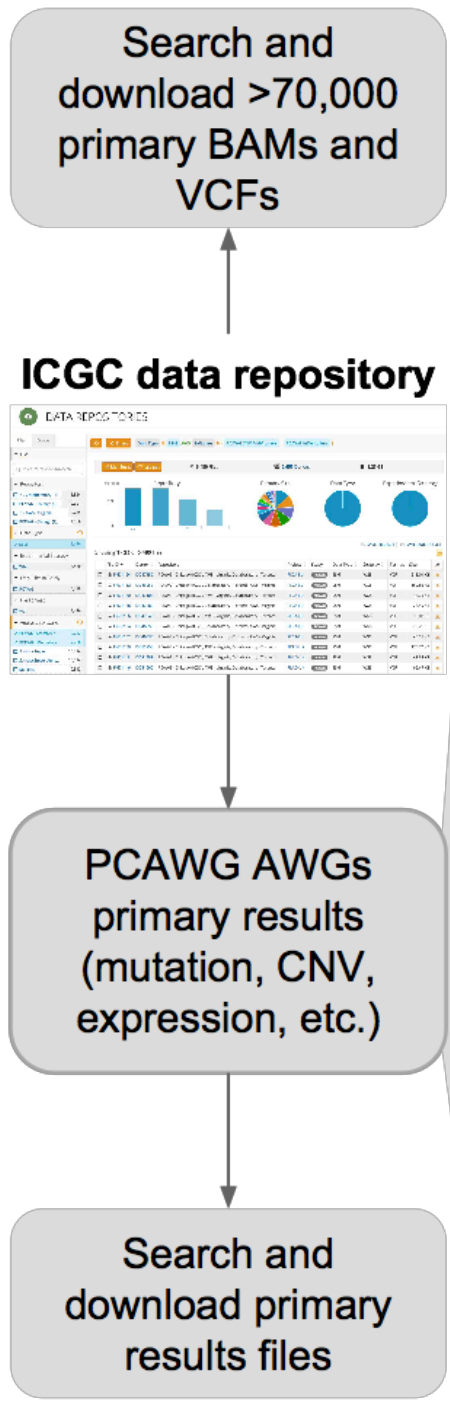

\section{Online resources for PCAWG data visualization}

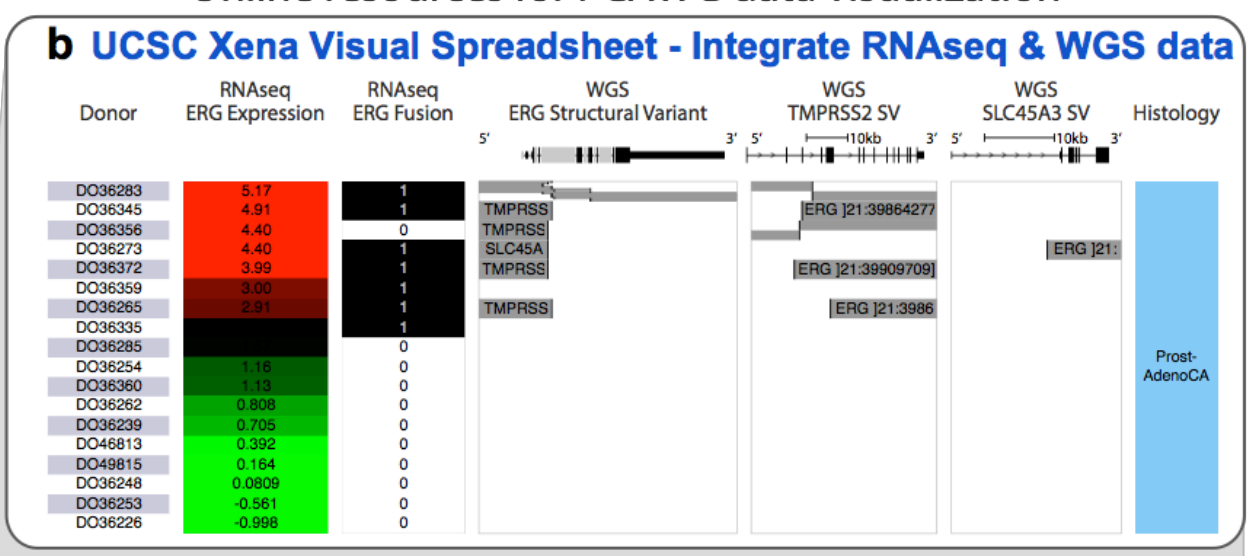

C Chromothripsis Explorer - Donor D036283

๑๑
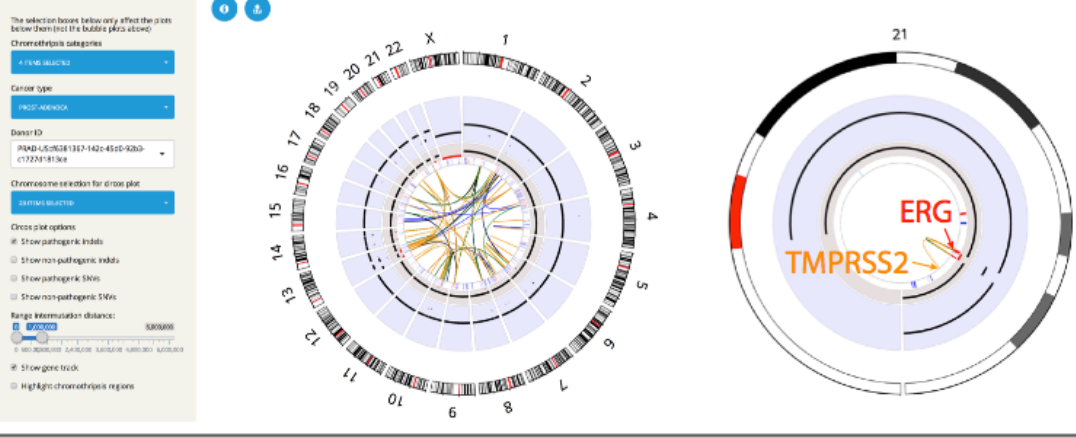

d Expression Atlas - Compare baseline vs. tumor expression
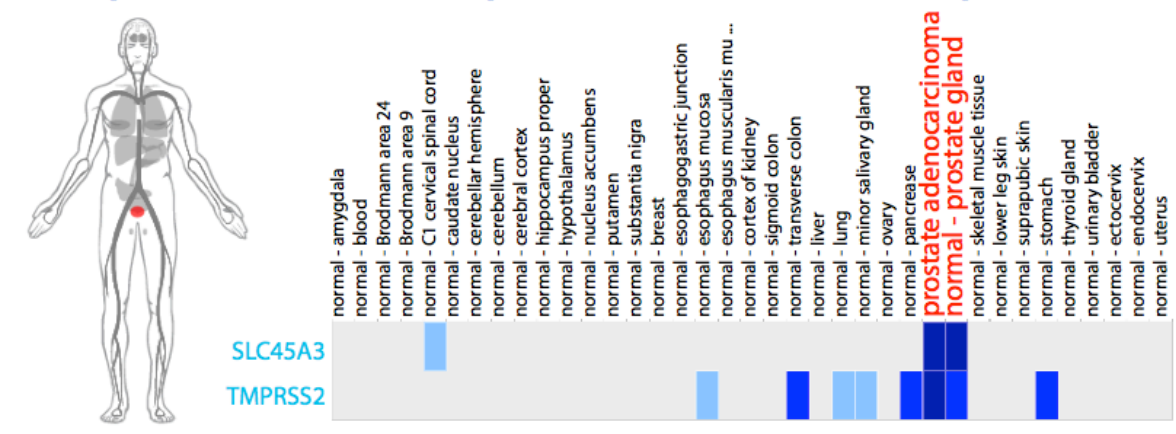

PCAWG-Scout - Mutual exclusivity and 3D mutation clustering

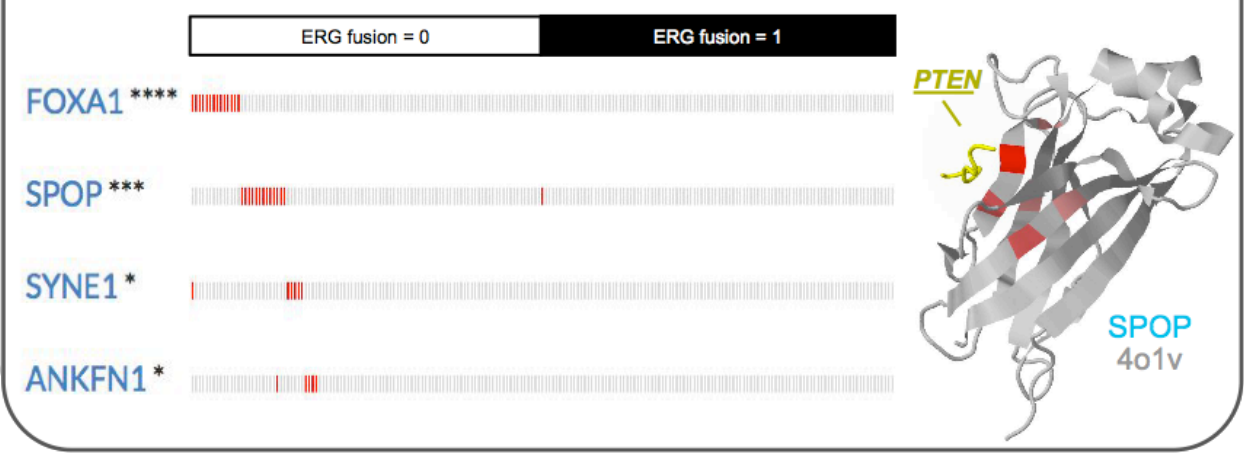


Figure 1. Online resources for PCAWG data download and visualization. These resources provide complementary views and analyses, illustrated by the example of mutually exclusive, recurrent events (such as ERG fusions and mutations in SPOP) detected in the prostate adenocarcinoma cohort.

(a) All PCAWG BAMs, VCFs, and analysis working group primary results files are available through the ICGC data portal's Data Repository tool (https://tinyurl.com/yburnde5). To obtain these files, the user downloads a manifest of selected files and then downloads the actual data files (with authorization if needed) using the ICGC download tool. UCSC Xena, Chromothripsis Explorer, Expression Atlas and PCAWG-Scout have each pre-processed the same primary analysis working group results files.

(b) The UCSC Xena Visual Spreadsheet shows that the ERG fusion is present in 8 out of 18 PCAWG prostate adenocarcinoma samples (https://tinyurl.com/y78adbl5), as detected by the PCAWG RNA-seq and wholegenome sequencing data. Each row corresponds to a sample. Columns, starting at the left, correspond to histology, ERG gene expression, and ERG fusion based on RNA-seq data (merged fusion calls from two methods (PCAWG Transcriptome Core Group 2018). The next three columns show structural variant calls (consensus calls by four methods Li 2017) using whole genome DNA-seq data for ERG, TMPRSS2, and SLC45A3. These data show that TMPRSS2 and SLC45A3 are fusion partners for ERG, and that the fusions correlate with over-expression of ERG. Fusions detected by RNA-seq and whole-genome sequencing are not always consistent. Here, even using a consensus of detection methods, one fusion detected by a consensus of RNA-based detectors is missed, and the converse is also seen. This example shows that an integrated visualization across multiple data types and algorithms provides a more accurate model of a genomic event.

(c) Chromothripsis Explorer provides an in-depth genome-wide view of the copy number alterations and structural variations in the 8 tumors with ERG fusion listed in B. For example, in tumor DO38283, chromosome 21 harbors multiple SVs that link it with chromosomes 2, 9, 13 and 21 (right). A closer look at the intrachromosomal SVs in chromosome 21 (left) reveals that the oncogenic fusion was generated by a deletion at chr21:39,988,805-40,578,907. Details about the total and minor copy number, as well as the SVs, can be obtained by hovering over these elements in the interactive version of the Circos plot. Circos plot visualizations for the other 7 donors are given in Supplementary Figure 6.

(d) The Expression Atlas shows a heatmap of genes (rows) and tissue or disease type (columns). Here we show the expression of ERG, SLC45A3, and TMPRSS2 in healthy human tissue (top heatmap), as derived from our re-analysis of the GTEx data set. The bottom heatmap shows expression in PCAWG data (https://tinyurl.com/y9fefymf). The human figure, also know as an anatomogram, shows the highlighted prostate tissue. We can see that TMPRSS2 and SLC45A3 show very high prostate-specific expression. Both genes have been used by the tumor to fuse its promoter to ERG, driving ERG over-expression.

(e) PCAWG-Scout complements the above analysis by identifying recurrent mutational events in tumors without ERG fusion (fusion = 0). On the left, PCAWG-Scout shows a mutation exclusivity analysis (using Fisher's exact test), which identifies FOXA1 $\left({ }^{* * *},<0.0001\right)$, SPOP $\left({ }^{* * *},<0.001\right)$, SYNE1 $\left({ }^{*},<0.05\right)$, and ANKFN1 $\left({ }^{*},<0.05\right)$ as significantly associated with non-fusion tumors (https://tinyurl.com/ybqpou52). In the 3D protein structure of SPOP shown on the right, mutations are seen to cluster tightly around the region that overlaps with the interaction surface of PTEN. The portion of PTEN that interacts with SPOP is shown in yellow, along with the SPOP structure. Red indicates recurrent mutations in SPOP with a brighter red indicating higher rate of recurrence.

Methods to reproduce views in $\mathbf{a}, \mathbf{b}, \mathbf{c}, \mathbf{d}$ and $\mathbf{e}$ are available in the Supplementary Information. 
$\mathbf{a}$

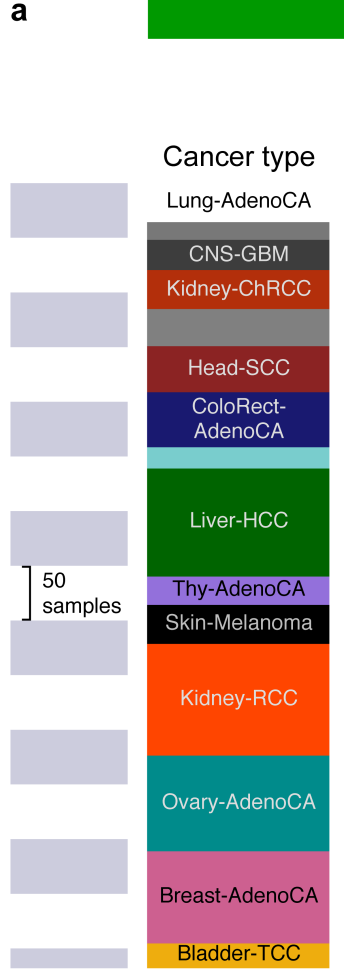

Public hub data

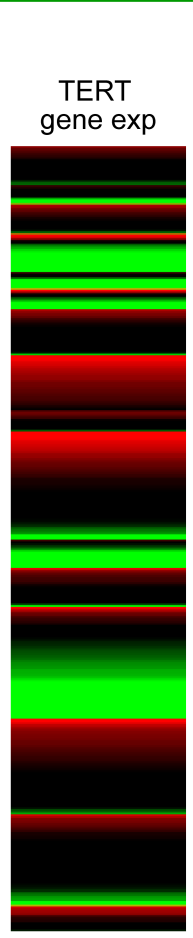

$-9.0 \sim 1.2$ $\log 2$ (FPKM-uq)

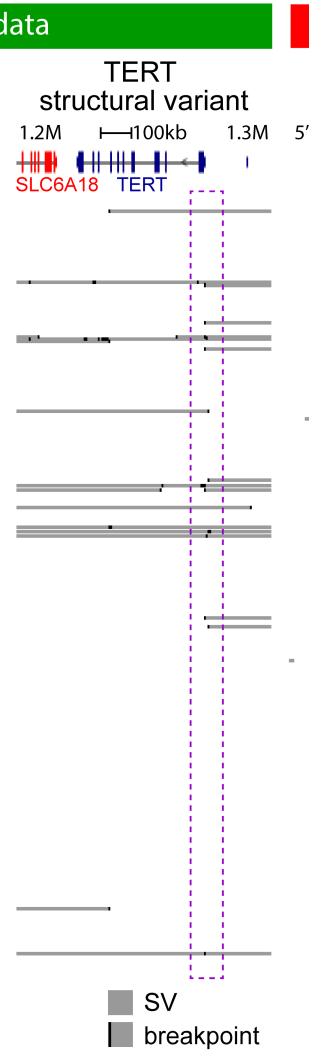

\section{Local hub data}

TERT simple mutation

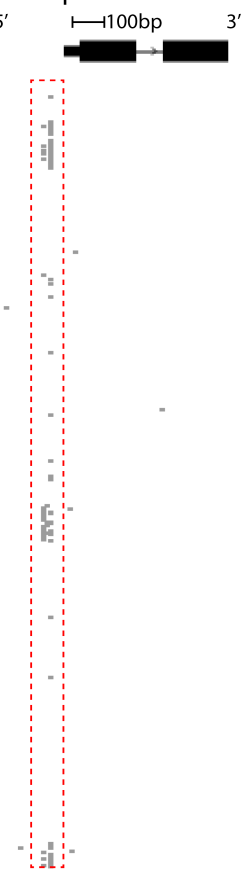

mutation

\section{Derived data}

TERT

alteration type

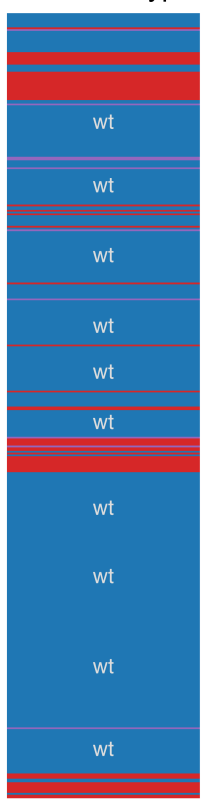

wt SV

Promoter mutation b

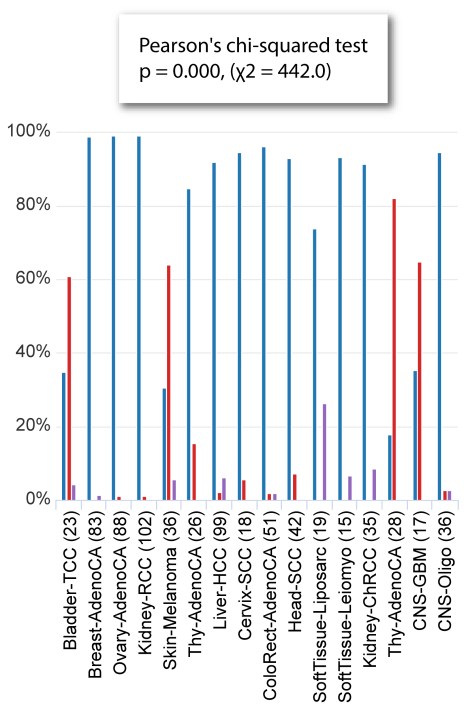

TERT alteration type

TERT wt

TERT promoter mutation

TERT structural variant

Figure 2. Xena Views of TERT across cancer types. (a) Visual Spreadsheet view of TERT multi-omics data across PCAWG cancer types. Data from the PCAWG public hub are under the green section, protected data from the user's local Xena hub is under the red section. Data is integrated in the browser, keeping private data protected. Even though the data is distributed across multiple hubs and those hubs have different access control, it appears to the user to come from a unified dataset, allowing easy visualization and data integration. We can see that many cancer types have TERT alterations either as simple somatic mutations in the promoter region, as seen in the pileup highlighted in the red box, or as structural variants, as seen in the breakpoint pileup upstream of TERT highlighted in the purple box. The last column was dynamically made in the browser showing which samples have promoter mutation, which have structural variants, and which have none. No sample is observed to have both promoter mutations and structural variants, as these two types of alterations are mutually exclusive. (b) Distribution of different types of TERT alterations across cancer types, as shown in Xena chart view. Xena automatically runs the appropriate statistical test for every chart. Here we can see that there is significant difference in the type of alteration in different cancer types. Cancer types that do not have TERT alterations in the PCAWG data are excluded from both (a) and (b) views. 

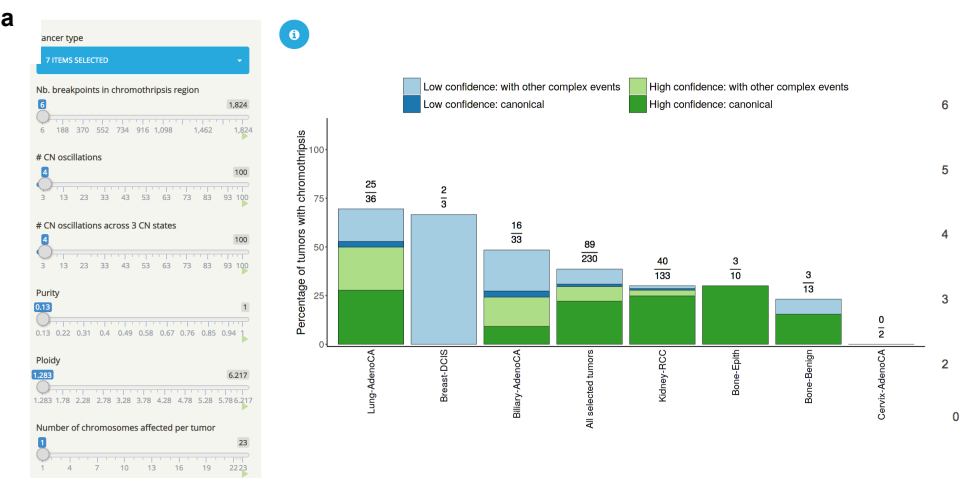

b

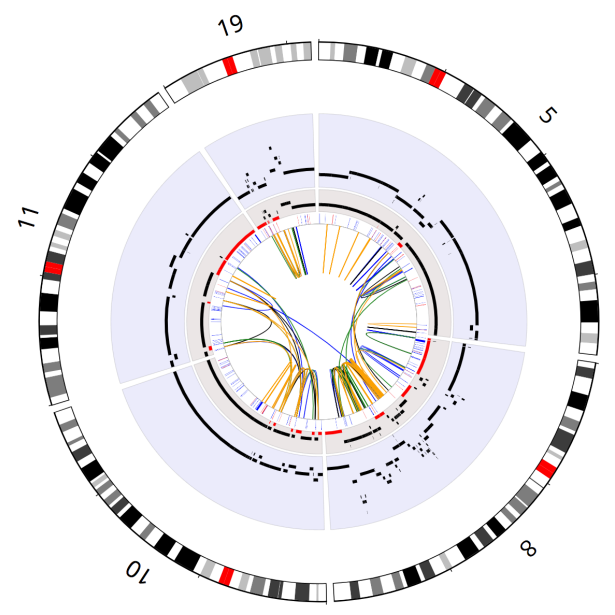



- Highlight chromothripsis regions

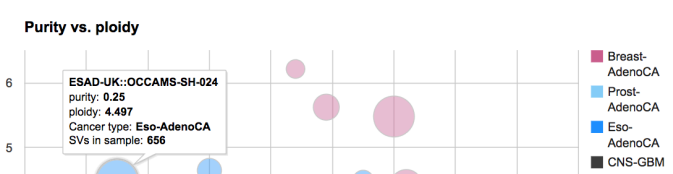

- CNS-GBM

Figure 3. Functionalities of Chromothripsis Explorer. (a) Interactive barplot for the visualization of chromothripsis rates for selected cancer types. The left-hand side panel corresponds to variables used for the detection of chromothripsis patterns (e.g., number of copy number oscillations; Cortes-Ciriano 2018). The user can modify the values of these to explore chromothripsis rates across variable stringency criteria. The righthand side panel illustrates additional functionalities to explore the relationship between purity and ploidy for tumors from selected cancer types. (b) Visualization of complex rearrangements involving 5 chromosomes in a ColoRect-AdenoCA patient (ICGC ID: D09034). The right-hand panel shows a zoomed view of chromosome 8 that illustrates the tracks available in the Circos plots. From the outer to the inner ring, the tracks correspond to hg19 cytobands, SNVs (colored according to the mutation type and distributed according to the inter-mutation distance), total copy number over a blue background, minor copy number (LOH regions, i.e., with a minor copy number of equal to 0 , are depicted in red), gene track, and SVs. Further information about the tracks can be accessed by clicking on the blue information circle located above the Circos plot. 


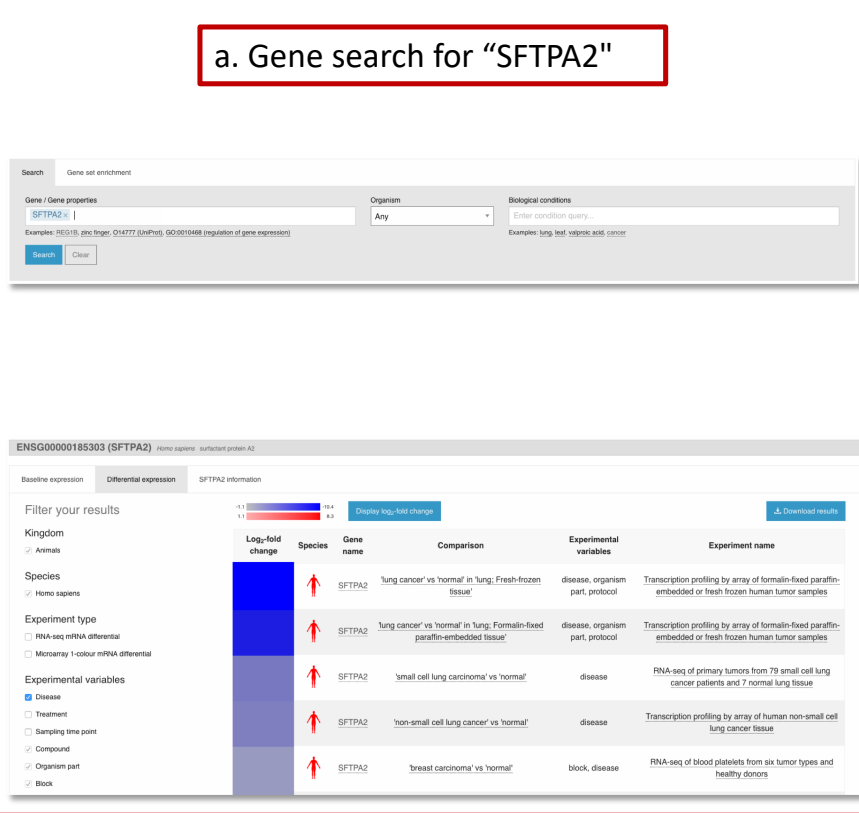

d. "SFTPA2" is downregulated in lung cancers in other studies within Expression Atlas b. "SFTPA2" is highly expressed in lung tissue

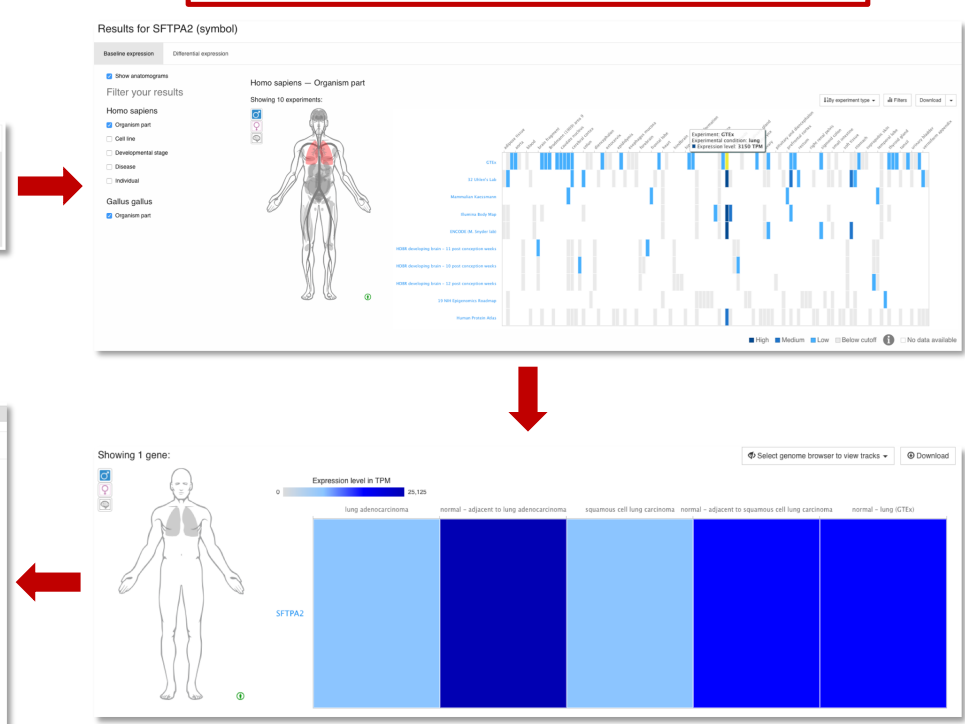

c. "SFTPA2" has lower expression value in lung cancer types than healthy tissue (PCAWG data)

Figure 4. Example gene search in Expression Atlas. (a) Searching for experiments where SFTPA2 is expressed or differentially expressed. (b) Viewing expression of SFTPA2 in different tissues, across all baseline experiments, shows a consistent, high expression in lung. (c) Looking for the same gene in the PCAWG study within Expression Atlas, we see that it displays low expression in lung cancers (lung adenocarcinoma and squamous cell lung carcinoma), whereas it is highly expressed in normal tissue whether it is adjacent to lung adenocarcinoma or adjacent to squamous cell lung carcinoma. It is also highly expressed in lung samples from GTEx. (d) Finally, through the pages of differential assays in Expression Atlas, we confirm that SFTPA2 is down-regulated in further studies of lung cancer in Expression Atlas. 


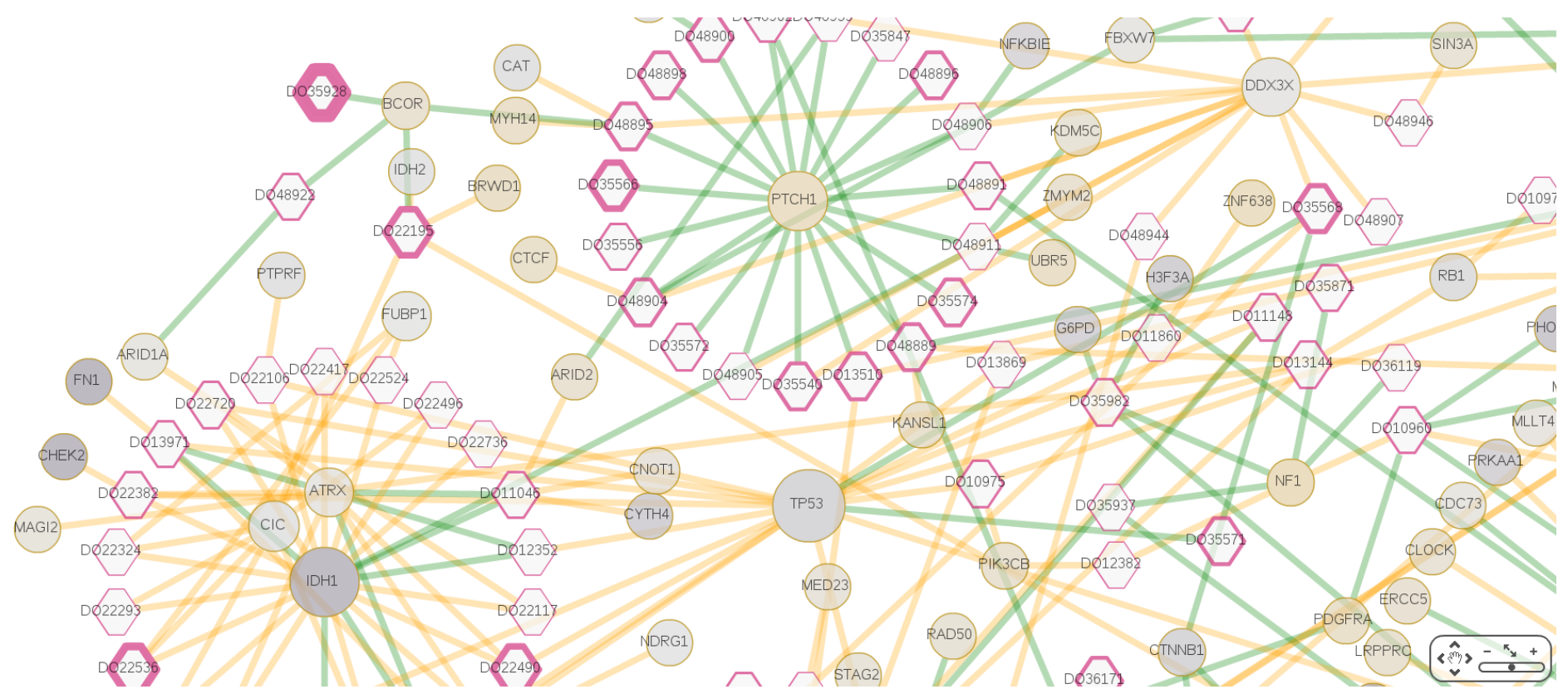

Figure 5. Customized visualization of the CNS meta-cohort donor driver events. This cytoscape-based visualization, available from the Study entity report page for the cohort, shows donors as hexagons and genes as circles. Edges represent driver events predicted (orange) and validated (green); donor driver events are curated by the drivers working group. Driver events for IDH1 are mostly predicted while for PTCH1 all are validated. The border size of the donors is their reported survival time. The size of the gene nodes is proportional to the significance of the extent to which mutations over these genes in this cohort are more damaging than expected; IDH1, TP53 and DDX3X stand-out. The color of the genes is proportional to their differential expression t-value statistic when comparing tumor samples with IDH1 mutations with the remaining tumor samples. The color is a gradient from repressed (dark purple) and overexpressed (gold) genes, with an intermediate color for non-deregulated (light grey); IDH1 is among the most repressed, along with FN1 and CHEK2, which have driver events co-occurring with IDH1 on some samples, while PTCH1 is overexpressed on IDH1 mutants. The extreme values for the gradient are taken genome wide and not limited to the genes present in the graph. Graphical aesthetics of node border-width, node size, node color, and edge color are configured interactively from data pulled from tables while exploring the network of linked reports around this cohort. Methods to reproduce this visualization are available in the Supplementary Information. 


\section{References}

Adamo, P., Ladomery, M. R. The oncogene ERG: a key factor in prostate cancer. Oncogene 35, 403414 (2016).

Baca, S. C., Prandi, D., Lawrence, M. S., Mosquera, J. M., Romanel, A., et al. Punctuated evolution of prostate cancer genomes. Cell 153, 666-77 (2013)

Campbell, P. J., Getz, G., Stuart, J. M., Korbel, J. O., Stein, L. D., et al. Pan-cancer analysis of whole genomes. Preprint at https://www.biorxiv.org/content/early/2017/07/12/162784 (2017).

Carithers, L. J., Ardlie, K., Barcus, M., Branton, P. A., Britton, A., et al. A Novel Approach to HighQuality Postmortem Tissue Procurement: The GTEx Project, Biopreservation and Biobanking 13, 311-319 (2015).

Chase, A. M., Yi, Q., DiSera, T., D'Astous, B. \& Marth G. T. bam.iobio: a web-based, real-time, sequence alignment file inspector. Nature Methods 11, 1189-1189 (2014).

Cortes-Ciriano, I., Lee, J., Xi, R., Jain, D., Jung, Y. L., et al. Comprehensive analysis of chromothripsis in 2,658 human cancers using whole-genome sequencing. Preprint at https://www.biorxiv.org/content/early/2018/05/30/333617 (2018).

Goldman, M., Craft, B., Kamath, A., Brooks, A.N., Zhu, J., et al. The UCSC Xena Platform for cancer genomics data visualization and interpretation. Preprint at https://www.biorxiv.org/content/early/2018/08/28/326470 (2018)

Kent, W. J., Sugnet, C. W., Furey, T. S., Roskin, K. M., Pringle, T. H.,et al. The human genome browser at UCSC. Genome Res 12, 996-1006 (2002).

Korbel J.O., \& Campbell P.J. Criteria for inference of chromothripsis in cancer genomes. Cell 152, 1226-36 (2013)

Li, Y., Roberts, N., Weischenfeldt, J., Wala, J. A., Shapira, O., Schumacher ,S., et al. Patterns of structural variation in human cancer. Preprint at https://www.biorxiv.org/content/early/2017/08/27/181339 (2017).

Melé, M., Ferreira, P. G., Reverter, F., DeLuca, D. S., Monlong, J., et al. The human transcriptome across tissues and individuals. Science 348, 660-665 (2015).

Notta, F., Chan-Seng-Yue, M., Lemire, M., Li, Y., Wilson, G.W., et al. A renewed model of pancreatic cancer evolution based on genomic rearrangement patterns. Nature 538, 378-382 (2016) 
PCAWG Transcriptome Core Group, Calabrese, C., Davidson, N. R., Fonseca, N. A., He, Y., et al. Genomic basis for RNA alterations revealed by whole-genome analyses of 27 cancer types. Preprint at https://www.biorxiv.org/content/early/2018/03/12/183889 (2018).

Petryszak, R., Keays, M., Tang, Y. A., Fonseca, N. A., Barrera, E., et al. Expression Atlas update--an integrated database of gene and protein expression in humans, animals and plants. Nucleic Acids Research 44, D746-D752 (2016).

Piñeiro-Yáñez, E., Reboiro-Jato, M., Gómez-López, G., Perales-Patón, J., Troulé, K., et al. PanDrugs: a novel method to prioritize anticancer drug treatments according to individual genomic data. Genome Medicine 10, 1 (2018).

Sabarinathan, R., Pich, O., Martincorena, I., Rubio-Perez, C., Juul, M., et al. The whole-genome panorama of cancer drivers. Preprint at https://www.biorxiv.org/content/early/2017/12/23/190330 (2017).

St John, J., Powell, K., Conley-Lacomb, M. K., Chinni, S. R. TMPRSS2-ERG Fusion Gene Expression in Prostate Tumor Cells and Its Clinical and Biological Significance in Prostate Cancer Progression. Journal of Cancer Science and Therapy 4, 94-101 (2012).

Stephens, P. J., Greenman, C. D., Fu, B., Yang, F., Bignell, G. R., et al. Massive genomic rearrangement acquired in a single catastrophic event during cancer development. Cell 144, 27-40 (2011)

Yu, Y., Ouyang, Y., Yao, W. shinyCircos: an R/Shiny application for interactive creation of Circos plot. Bioinformatics 34, 1229-1231 (2018).

Yung C. K., O'Connor B. D., Yakneen, S., Zhang, J., Ellrott, K., et al. Large-Scale Uniform Analysis of Cancer Whole Genomes in Multiple Computing Environments. Preprint at https://www.biorxiv.org/content/early/2017/07/10/161638 (2017).

Zerbino, D. R., Achuthan, P., Akanni, W., Amode M. R., Barrell D., et al. Ensembl 2018. Nucleic Acids Research, 46, D754-D761 (2018). 